\title{
Creating complex social conjunction categories from simple categories
}

\author{
REID HASTIE \\ University of Colorado, Boulder, Colorado \\ COLIN SCHROEDER \\ SYSTAT Corporation, Evanston, Illinois \\ and \\ RENÉE WEBER \\ Leo Burnett Company, Chicago, Illinois
}

\begin{abstract}
College student subjects responded to simple or conjunction social category labels (e.g., "mechanics"; "female mechanics") in two experiments. In the first experiment, they listed attributes that they believed described a typical member of the category and, in the second experiment, they rated the characteristics of typical members of the category on 15 trait-adjective rating scales. A large proportion of the subjects' responses to conjunction categories in the attribute-listing task involved the production of emergent attributes that had not been mentioned for either of the ingredient categories. Similarly, a large proportion of trait-adjective ratings for the conjunction categories fell outside the bounds defined by the ratings of the simple ingredient categories. These two findings, along with the subjects' retrospective explanations for their ratings, suggest that people frequently engage in a complex reasoning process in order to infer the attributes of a conjunction category that is defined by the intersection of two simple social categories. A process model is outlined to account for the subjects' attribute-listing and rating responses.
\end{abstract}

For several years, we have been involved in research to examine the processes that occur when people respond to the dilemmas posed by receiving apparently conflicting information about another person. Some of this research has utilized a traditional impression-formation task, in which the subject receives pieces of information about another person in the form of trait adjectives, sentences describing behavior, or short film clips that illustrate behaviors (e.g., Hastie \& Kumar, 1979). When a piece of information occurs that "contradicts" the general impression implied by the preponderance of the information, subjects respond by engaging in a more complex, "creative" reasoning process, the primary goal of which is to explain why the unexpected event has occurred.

We have also studied how individuals and small groups resolve the dilemma posed by conflicting claims in legal testimony about events that have occurred in social situations such as disputes, physical confrontations, or robberies (see Hastie, Penrod, \& Pennington, 1983). Again, when contradictory, apparently inconsistent information appears, people respond to it in a more complex, "deeper" manner, by attempting to construct a coherent "story" of the

The authors would like to thank Thomas Ostrom, Nancy Pennington, Lance Rips, and Edward Smith for their valuable advice concerning this research. Support for the research was provided by NSF Grant BNS8717259. Requests for reprints should be addressed to Reid Hastie, Center for Research on Judgment and Policy, Campus Box 344, University of Colorado, Boulder, CO 80309-0344. events that can resolve the conflicting implications of the evidence (see Pennington \& Hastie, 1986).

In the present report, we summarize the results of some preliminary investigations of the manner in which people reason about general social categories. The focal question in the research concerns how people construct a representation of a complex conjunction category from two simpler ingredient categories. We were particularly interested in how this process would proceed when the two ingredient categories evoked apparently inconsistent or contradictory implications about the attributes of a typical member of the conjunction category.

We will use the example of our subjects' inferences about the attributes of a member of the category "female mechanics" to illustrate our methods and findings. Subjects' ratings were highly discrepant for the two simple categories "female" and "mechanics" on several trait-adjective rating scales-namely, dimensions labeled warm-cold, intelligent-unintelligent, lower class-upper class, strong-weak, active-passive, and dominant-submissive. Thus, preliminary ratings of the simple category attributes led us to expect that for a combination like "female mechanic," subjects would be faced with some conflicting implications from the ingredient categories when attempting to list attributes of the conjunction category.

The literature on impression formation and on category representation suggests two types of models that might be relevant to the task of predicting the attributes of the complex conjunction from the ingredient-category attri- 
butes. First, considerable research on social judgment suggests that a candidate model would be some type of weighted average combination rule that would take scale values for the relevant attributes from the two simple categories and combine them into a compromise or an average value for the conjunction (Anderson, 1981).

A second candidate model comes from research by Smith, Osherson, Rips, and Keane (1988), who have proposed a selective modification model to predict the attributes of categories created by modifying a noun with an adjective (e.g., "green apple," "'round vegetable," etc.). Noting that the selective modification model has not been proposed for exactly the case we consider here (two nouns conjoined), the type of attributes that are predicted for the conjunction from a model of this type would be limited to attribute values selected on or between the ingredientcategory values.

Without getting too far ahead of our story, we anticipated that the occurrence of emergent attributes-attributes not mentioned for either of the simple ingredient categories, but cited in descriptions of the conjunction-would be especially likely to occur for the conflicting ingredient conjunction pairs. Furthermore, we expected that ratings of attribute values on trait-adjective scales that were "outside the bounds" defined by the two ingredient-category ratings would be especially likely to occur for conflicting combinations. Either of these types of results, emergent attributes or outside ratings, would be impossible to account for with reference to simple forms of the weighted averaging and selective modification models.

\section{STUDY 1}

An attribute-listing task has been used frequently in research on category representation to elicit an initial "snapshot" of the contents of a subject's mental representation of a category. In our first study, subjects were asked to list the attributes that came to mind when they thought of either a simple (e.g., "mechanics") or a conjunction (e.g., "female mechanics") category. One focus of the analysis was to detect the rate at which emergent attributes occurred in the protocols. An emergent attribute was an attribute that had not been listed for either of the simple categories but was listed for the conjunction.

\section{Method}

Subjects. One hundred and forty undergraduate introductory psychology students participated in the experiment in order to meet a course requirement for research participation. The experimental session lasted approximately $30 \mathrm{~min}$.

Procedure. Each subject was presented with a triplet of category terms (e.g., "females," "'mechanics," “female mechanics”). For each category, the subject was given the following instruction: "In the space below, make a list of the attributes or characteristics that you think would describe a typical member of the category of [female mechanics].",

Materials. Three sets of four social category labels were used to create the experimental materials. The labels were selected so that when three $2 \times 2$ tables were created by pairing individual terms, two of the pairs would describe relatively rare, incongruent con- junction categories, and two of the pairs would describe relatively common conjunctions. For example, the labels "male" and "female" were paired with "mechanic" and "nurse" to create the four conjunctions: "male mechanic" and "female nurse" (common) and "male nurse"' and "female mechanic" (incongruent). The three sets of four labels are displayed in Table 1 . These category labels were selected to provide conjunctions that were truly composed by the intersection set of members belonging to both the first ingredient category and the second ingredient category. These intersection conjunctions can be contrasted with complex conjunctions, which were not studied in the present research, in which one term serves to modify the meaning of the other term in a strong linguistic sense (e.g., "criminal lawyer"; Murphy, 1988).

\section{Results}

Subjects generated between 4 and 15 attributes for the ingredient and conjunction categories. On the average, the most attributes were generated for the occupation label categories (e.g., for "mechanics," mean number of attributes $=9.3$ ); the next most for the background categories (e.g., "females," mean attributes $=6.4$ ); and the fewest for the conjunction (e.g., "female mechanics," mean attributes $=6.2$ ). We were especially interested in the rate at which emergent attributes were generatednamely, attributes that were not listed for the ingredient categories but were listed for the conjunction. We used a strict definition of "emergent": if for the conjunction a subject used a new word that had not appeared in the descriptions of either of the ingredient categories, the conjunction was credited with one emergent attribute. This meant that unless the new attribute was virtually identi$\mathrm{cal}$ in its root word to the wording in the ingredient category, it was treated as emergent (e.g., "muscles" vs. "muscular" would be counted as a repetition, but "strong"' vs. "muscular" would be counted as an emergent attribute).

Table 1 lists the proportions of emergent attributes that were generated by subjects responding to the 12 conjunction categories. Two characteristics of the figures in the table are worth noting: First, there are many emergent attributes; in some cases, more than $40 \%$ of the attributes generated for a conjunction had not been cited for either of the ingredient categories. Second, the emergent attributes are likelier to occur for the conjunctions that we labeled incongruent than for the common

Table 1

Proportions of Emergent Attributes

\begin{tabular}{lcc}
\hline & Mechanic & Nurse \\
\cline { 2 - 3 } Male & .18 & .42 \\
& .34 & .07 \\
Blackale & Janitor & Banker \\
Jewish & .21 & .40 \\
& .34 & .27 \\
Feminist & Social Worker & Bank Teller \\
Republican & .08 & .19 \\
\hline
\end{tabular}


Table 2

Example Attribute Ranking Task

Make a slash mark through each scale below to indicate the degree to which the typical member of the category FEMALE MECHANICS possesses the relevant quality or trait.

\begin{tabular}{|c|c|}
\hline Ambitious & Unambitious \\
\hline Warm & Cold \\
\hline Hostile & Friendly \\
\hline Introverted & Extroverted \\
\hline Intelligent & Unintelligent \\
\hline Lower class & Upper class \\
\hline Likeable & Unlikeable \\
\hline Adventurous & Cautious \\
\hline Honest & Dishonest \\
\hline Calm & Anxious \\
\hline Strong & Weak \\
\hline Active & Passive \\
\hline Dominant & Submissive \\
\hline Imaginative & Unimaginative \\
\hline onscientious & Careless \\
\hline
\end{tabular}

conjunctions. The off-diagonal values in the tables (e.g., "female mechanic," "male nurse") are larger than the main-diagonal values (e.g., "female nurse," "male mechanic"').

We believe that the high rate of emergent attributes is a signal that subjects are doing something special to deal with the construction of an uncommon or novel conjunction. But before we turn to a discussion of what that "something special" might be, we want to present the results of a second study with a different method that converges on the conclusions of the first study.

\section{STUDY 2}

Although the results of the attribute-listing task are suggestive, they are far from compelling evidence for process differences in responses to common as opposed to uncommon conjunctions. One major interpretive problem arises from the experimenter's decision about what an appropriate criterion to admit an attribute as emergent is, as opposed to considering it simply as a synonymous repetition of an attribute that has been listed for one of the ingredient categories. Obviously, the "strict" criterion used in Study 1 will favor the identification of even very similar attributes as "emergent." In a second study, we presented subjects with the same materials; but this time, rather than list attributes, they were asked to express their judgments of the simple ingredient categories and the conjunction categories by making ratings on bipolar traitadjective rating scales.

\section{Methods}

Subjects. Ninety-eight undergraduate students served as subjects in a 30-min experimental session for which they were rewarded with credit toward their introductory psychology course requirements.

Procedure. The subjects were instructed as follows: "Make a slash mark through each scale below to indicate the degree to which the typical member of the category [female mechanics] possesses the relevant quality or trait." This instruction was followed by 15 bipolar rating scales labeled at each end with opposing trait adjec- tives (see Table 2 for the labels on the 15 scales). After making ratings on the adjective rating scales for two simple categories (e.g., "female" and "mechanic"), the subjects were asked to make the same ratings for the conjunction category (e.g., "female mechanic").

After the rating task was over, a 10-min break was provided; then, an experimenter discussed each of the ratings with the subject. The subjects were asked to reflect retrospectively on the reasons why they made the ratings as they did. For example, the experimenter might have initiated the discussion thus: "On the dominant-submissive scale, you gave a rating here for females, here for mechanics, and here for female mechanics. What were you thinking about when you made these ratings?" If the subject was silent following this probe, the experimenter would probe once more: "Can you tell me anything about what you were thinking about when you made these ratings?" If the subject was still unresponsive, the experimenter moved on to the next set of ratings.

\section{Results}

Rating scale responses. Subjects' ratings on the two ingredient categories and for the conjunction category on each of the 15 bipolar scales were tabulated and compared. The analysis focused on four categories of responses: (1) ratings for conjunctions that fell within plus-or-minus one scale unit (on a 10-point scale) of the occupation rating; (2) ratings for conjunctions that fell within plus-orminus one scale unit of the subject's rating for the simple background category (ethnicity or gender) on the trait; (3) ratings for conjunctions that fell between the background and occupation ratings; and (4) conjunction ratings that fell outside the bounds defined by the range of the ratings for the occupation and background. We were especially interested in these outside ratings. We consider them similar to the emergent features in the attributelisting task; an outside rating indicates that the subject has gone "out of the bounds" of values defined by the two ingredient categories, in a manner analogous to generating a novel attitude "outside" the meanings of the ingredient-category attributes.

Overall, .36 of the ratings were on the occupation value (plus-or-minus one); .11 were within one scale unit of the background factor; .25 fell somewhere between the background and occupation ratings; and .28 were outside the range defined by the two ingredient ratings. Table 3 provides a summary of the proportions of ratings subjects made that were outside the range defined by the occupation and background values. Again, we can see that out-

Table 3

Proportions of Outside Ratings
Male

Female

\begin{tabular}{cc} 
Mechanic & Nurse \\
\hline .22 & .36 \\
.37 & .25
\end{tabular}

Black

Jewish

\begin{tabular}{cc} 
Janitor & Banker \\
\hline .20 & .40 \\
.35 & .24
\end{tabular}

Feminist

Republican
Social Worker

26

30
Bank Teller 
side ratings are frequent; as high as .40 for one of the 12 conjunctions. And, again, we see the pattern of the off-diagonal, incongruent conjunctions receiving substantially more of the outside ratings than the common maindiagonal conjunction cases.

Retrospective explanations. The tape-recorded explanations that subjects provided for their ratings were content-analyzed to see if they would yield any clues about the processes in which subjects were engaged when they reasoned about conjunction categories. Table 4 is a summary of the content coding scheme that was applied to these retrospective reports. The "no comment" category is composed mostly of responses in which the subjects simply repeated their ratings, but did not provide a substantive explanation. The two most frequent types of responses were references to the background characteristics of the person being rated or to the person's occupation to provide an explanation for the conjunction rating. It is also important to notice that in very few cases did subjects refer to a specific instance of a member of the category when they made their conjunction ratings. For our college student subjects, almost all of the references to specific instances were applied to conjunctions involving the "janitor" category.

About $10 \%$ of the responses fell into categories that we thought revealed more complex, deeper forms of reasoning about the conjunction. The most common of these were explanations for the rating that cited conditions necessary to enter the occupation. For example, "Well, you see, you've got a woman here with all these men. She's going to have to be a little more dominant if she's going to survive. It's just what it takes if you're going to become a woman in a man's job, not just anyone can take it." A second complex explanation involved references to the effects of being in an occupation on a person with distinctive background characteristics. For example, "As far as maybe being outgoing and doing things and active when they're with their customers. It's just something they have to do; to overcompensate and be a lot more active. They have to kind of prove themselves. They have a lot more to prove than a man in the same job." Two final complex categories were rarely applied, involving references to a contradictory combination of ingredients or references to a special subtype of the category created by the combination. Contradictory

Table 4

Think-Aloud Coding Scheme

\begin{tabular}{ll}
.20 & No comment \\
.02 & Reference to an instance \\
.18 & Background \\
.33 & Occupation \\
.14 & Blend of background and occupation \\
.06 & Condition to enter occupation \\
.04 & Effect of being in the occupation \\
.01 & Contradictory combination of background and occupation \\
.01 & Subtype created by background and occupation \\
.01 & Other \\
\hline
\end{tabular}

Table 5

Proportions of Conditions to Enter, Effect of Occupation, Contradictory Combination, and Subtype Explanations

\begin{tabular}{lcc}
\hline & Mechanic & Nurse \\
\cline { 2 - 3 } Male & .04 & .19 \\
& .17 & .09 \\
Female & Janitor & Banker \\
\cline { 2 - 3 } Jewish & .08 & .30 \\
& .19 & .04 \\
Feminist & & \\
Republican & Social Worker & Bank Teller \\
\hline
\end{tabular}

combination explanations occurred most frequently in reference to a discrepancy perceived between the values of a "social worker" and the values of a "Republican"; such a person was occasionally described as "screwed up and hard to predict." The male nurse category elicited most of the references to subtypes. For example, several subjects noted that male nurses would probably fill a special role, perhaps in a psychiatric or geriatric treatment facility, where their greater physical strength would be especially useful.

We were interested in whether or not these more complex types of explanations tended to be associated with the same conditions in which we have observed emergent features and outside ratings. Table 5 provides a summary of the conjunctions that elicited these four types of complex explanations and, again, it is clear that they occur most often for the uncommon conjunctions.

\section{DISCUSSION}

In this report, we have focused on three phenomena that appear when subjects reason about simple social categories and complex conjunction categories defined by noun-noun category intersections. First, we observed that, especially for apparently incongruent ingredient categories, members of conjunction categories were described by emergent attributes at a distinctively high rate. In some cases, more than $40 \%$ of the conjunction-category attributes had not been cited in the attribute lists for the simple, ingredient categories. Second, we observed a high proportion of trait-adjective rating scale values that occurred outside the bounds defined by the ratings (on the same attribute scales) for the simple ingredient categories. Essentially, we observed that the formation of some conjunctions, especially from conflicting simple categories, involved going beyond the rating scale values from the ingredient categories to find a plausible value for the conjunction. Third, the contents of think-aloud, retrospective explanations for the adjective scale ratings revealed a moderately high rate of references to complicated causal inferences to explain the conjunction-category ratings and their relation to the simple category ratings. More specifically, 
especially for the incongruent simple category pairs, conjunctions elicited references to conditions necessary for entrance into the conjunction category and to the effects of being in the conjunction category on the character of such a person.

These three results imply that simple models based on a weighted averaging rule or the selective modification "voting rule" would not be adequate to account for the manner in which subjects inferred the attributes of a rare conjunction.

We would like to end this paper on a speculative note by making some suggestions for the type of model that would be needed to account both for the distinctive emergent attribute and outside rating results and for the more typical compromise rating results.

First, Smith et al. (1988) have taken a step in the right direction by proposing that a frame structure characterizes the long-term memory representation for generic categories such as "female," "mechanic," and so forth. Such a frame would have a number of slots corresponding to the functions performed by a member of the category (e.g., associated goals, activities, "props," and locations); the conditions necessary for entrance into the category; the typical characteristics of members of the category (e.g., gender, race, social class, personality attributes); and pointers to subtypes of the category. Each slot would be associated with a central tendency-a default value for the relevant attributes-as well as with an expression of the variability in admissible values of a member of the category. In addition to these defaults and admissible values, there are, we believe, constraints-perhaps "soft constraints"-among attributes within the frame. For example, the values of activity slots within the function's subframe may constrain the expected personality values and also the values assigned to conditions for entrance into the category.

Given frame representations of simple categories, we believe that a two-stage model will be needed to account for the results of the rating and attribute-listing experiments. The first stage of this general model would proceed according to the types of compromising or averaging rules prescribed by the selective modification or weighted averaging models. However, at some point during the application of a modification or averaging process, it should be possible for that process to signal that it is "in trouble," perhaps because the default values expected on common attributes for the two ingredient-category frames are so discrepant that they signal a conflict or an impossibility.

The signal from the first-stage processes that a difficult or surprising condition has been encountered would lead to a second stage that would involve the construction of a more complicated solution to the conjunction-attribute inference problem. A careful (and imaginative) scrutiny of our subjects' think-aloud, retrospective explanations of their rating scale responses provides some clues about what this more complicated solution procedure might involve. Three general strategies seem to have been followed by subjects who produced outside ratings under conditions in which the two ingredient-category attributes were in conflict.

First, many subjects attempted to identify a relevant case or instance in long-term memory. Frequently this involved some analogical reasoning to find a match that was appropriate to, although not identical to, the experimenter's conjunction. For example: "I don't know a female mechanic but I know a construction worker. She's big, loud, uneducated...."

A second strategy appeared to rely on general rules abstracted from personal experience that had occurred before the experimental session; these rules were activated to solve the problem of inferring the conjunction attributes. For example: "A woman in a man's job has to be tough...." Or, "High achieving minorities tend to be very ambitious...."

A third strategy appeared that involved combining the constraints-often soft constraints-of many small ingredient premises, to make an inference about the characteristics of the conjunction. This strategy often took the form of a "mental simulation" process for the purpose of determining what kind of person it would take to perform the requisite activities of the occupation term in the conjunction. Subjects frequently reported images of what this person would look like. For example: "What kind of a woman would work in a garage ... she'd have to be tough, she'd be willing to fight for her rights ... I can see her working on a car and trading dirty jokes with the guy in the bay next to hers...."

We want to emphasize that these theoretical notes are an incomplete explanation of the results obtained in the present conjunction-category experiments. However, we think that to do justice to the contents of the subjects' think-aloud explanations, something along the lines we have sketched will be necessary. But we do not want to diminish the important contributions of the work of Smith et al. (1988) and the cognitive algebraists (e.g., Anderson, 1981), who have contributed much to our understanding of social inference processes. In fact, it is clear that our proposal of a second-stage process complements the contributions of these seminal theorists.

Research on the representation of social categories and the nature of inferences that are made to create descriptions of novel categories reveals complex judgment processes, in a manner in which the exploration of more commonplace, natural categories (e.g., fruits, vegetables, etc.) has not. Although emergent attributes and outside ratings have been observed when subjects reason about complex categories of a nonsocial type, the rate at which these distinctive phenomena occur is exceptionally high for the social categories we have studied. Reasoning about everyday social categories takes a complex form that challenges the predictive power of simple cognitive models 
that have been developed with less evocative stimulus materials.

\section{REFERENCES}

ANDERSON, N. H. (1981). Foundations of information integration theory. New York: Academic Press.

Hastie, R., \& KumaR, A. P. (1979). Person memory: Personality traits as organizing principles in memory for behaviors. Journal of Personality \& Social Psychology, 37, 25-34.
Hastie, R., Penrod, S. D., \& Pennington, N. (1983). Inside the jury. Cambridge, MA: Harvard University Press.

MurPhy, G. L. (1988). Comprehending complex concepts. Cognitive Science, 12, 529-562.

Pennington, N., \& Hastie, R. (1986). Evidence evaluation in complex decision making. Journal of Personality \& Social Psychology, 51, 242-258.

Smith, E. E., Osherson, D. N., Rips, L. J., \& Keane, M. (1988). Combining prototypes: A selective modification model. Cognitive Science, 12, 485-527. 